\title{
Precise laser spectroscopy of antiprotonic helium at CERN's Antiproton Decelerator
}

\section{Masaki Hori}

Published online: 5 March 2010

(C) The Author(s) 2010. This article is published with open access at Springerlink.com

\begin{abstract}
The ASACUSA collaboration of CERN has carried out several laser spectroscopy experiments of antiprotonic helium atoms. By comparing the results with three-body QED calculations, the antiproton-to-electron mass ratio was determined as $M_{\bar{p}} / m_{e}=1836.152674(5)$. We are now developing some laser systems and detectors to further improve the experimental precision.
\end{abstract}

Keywords Antiprotonic helium • CPT symmetry • Laser spectroscopy

\section{Introduction}

The ASACUSA collaboration at CERN has carried out several high-precision laser spectroscopy [1-4] experiments on antiprotonic helium atoms $\left(\bar{p} \mathrm{He}^{+} \equiv \bar{p}+\mathrm{He}^{2+}+\right.$ $\left.e^{-}\right)$since the construction of the Antiproton Decelerator (AD) facility in 1999. These atoms consist of a helium nucleus with an orbital electron in the 1s ground state, and the antiproton populating a Rydberg state with principal and angular momentum quantum numbers $n \sim \ell-1 \sim 38$. The atomic wavefunctions of these antiprotonic states have very little overlap with the helium nucleus, and so the antiproton can survive for many microseconds before annihilating through the strong interaction.

In the experiments described here, nanosecond lasers with MW-scale output energies were used to induce single-photon transitions of the antiproton in $\bar{p} \mathrm{He}^{+}$. These resonances were of the types $(n, \ell) \rightarrow(n-1, \ell-1)$ or $(n, \ell) \rightarrow(n+1, \ell-1)$ at

M. Hori

Max-Planck-Institut für Quantenoptik,

Hans-Kopfermann-Strasse 1, 85748 Garching, Germany

M. Hori (凶)

Department of Physics, University of Tokyo,

Hongo, Bunkyo-ku, Tokyo 113-0033, Japan

e-mail: Masaki.Hori@cern.ch 
Fig. 1 Energy level diagrams of $\bar{p}^{4} \mathrm{He}^{+}$and $\bar{p}^{3} \mathrm{He}^{+}$atoms in the region of principal and angular momentum quantum numbers $n \sim l-1 \sim 31-40$. The antiprotons cascade via radiative transitions of the type $(n, \ell) \rightarrow(n-1, \ell-1)$ which conserve the vibrational quantum number $v=n-\ell-1$. Wavelengths of the transitions studied by laser spectroscopy are indicated in nanometers

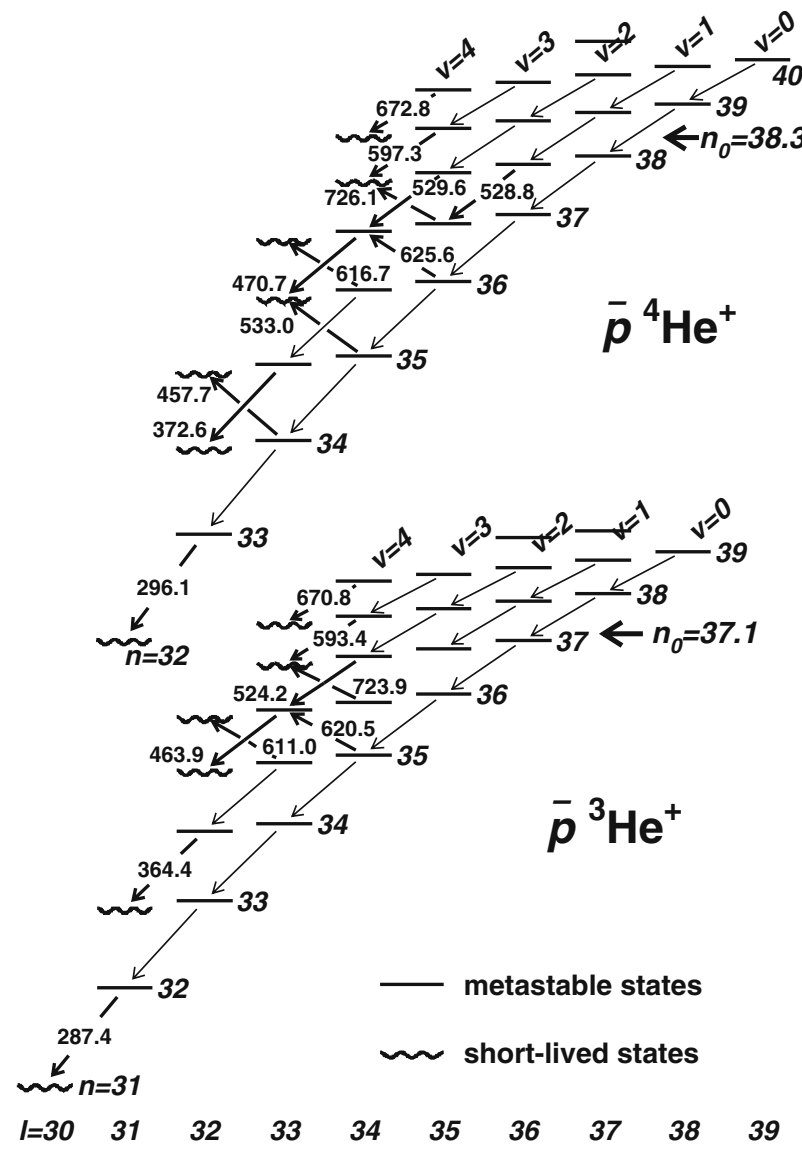

wavelengths $\lambda=264-726 \mathrm{~nm}$. By comparing twelve transition frequencies of $\bar{p} \mathrm{He}^{+}$ with the results of three-body QED [5-8] calculations, the antiproton-to-electron mass ratio was determined as $M_{\bar{p}} / m_{e}=1836.152674(5)$ [4, 9]. In this paper, we briefly summarize some of the technical aspects of these experiments, and recent developments in high precision lasers and detectors carried out by our group. ASACUSA is one of four atomic physics experiments [10-12] at the AD.

\section{Laser spectroscopy of antiprotonic helium}

The energy level diagrams of the $\bar{p}^{4} \mathrm{He}^{+}$and $\bar{p}^{3} \mathrm{He}^{+}$isotopes in the region $n=31-$ 40 and $\ell=30-39$ are shown in Fig. 1 . The atoms were synthesized in the following way. The AD provided pulsed beams of length $200-300 \mathrm{~ns}$ containing $\sim 2 \times 10^{7}$ antiprotons of energy $5.3 \mathrm{MeV}$ at a repetition rate of $0.01 \mathrm{~Hz}$. These antiprotons were decelerated to $\sim 100 \mathrm{keV}$ using a radiofrequency quadruple decelerator $[3,13]$, before being allowed to stop in a cryogenic helium target (Fig. 2). Some of the antiprotons replaced the electrons in normal He and were captured [14] into states with principal quantum number $n_{0}=\sqrt{M^{*} / m_{e}}$, wherein $M^{*}$ denotes the reduced 


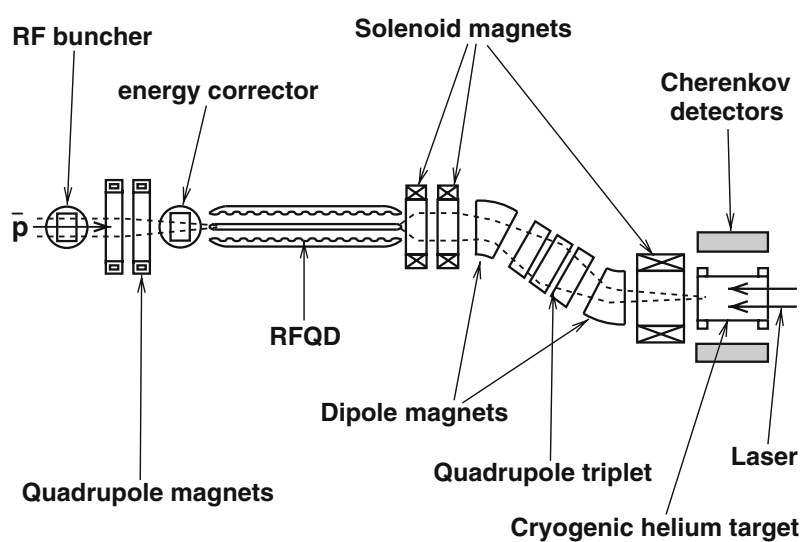

Fig. 2 Experimental layout for laser spectroscopy of antiprotonic helium. Dashed lines indicate trajectories of antiproton beam. The $5.3-\mathrm{MeV}$ antiprotons provided by AD are first decelerated to $\sim 100 \mathrm{keV}$ using a radiofrequency quadruple decelerator. The antiprotons are then transported by a momentum analyzer and stopped in helium target. The $\bar{p} \mathrm{He}^{+}$atoms synthesized in this way are irradiated by a pulsed laser beam. The charged pions emerging from antiproton annihilations in the target are detected by Cherenkov counters, see text

mass of the antiproton-helium nucleus system, and $m_{e}$ the electron mass. The antiproton cascaded [15-17] through a series of metastable states with microsecondscale lifetimes (indicated by solid lines in Fig. 1), making radiative transitions of the type $(n, \ell) \rightarrow(n-1, \ell-1)$ which conserve the vibrational quantum number $v=$ $n-\ell-1$.

We induced laser transitions [18] from these metastable states, to states with nanosecond-scale lifetimes (wavy lines) against Auger electron emission [19, 20]. Singly-charged, two-body $\bar{p} \mathrm{He}^{2+}$ ions [21] remained after Auger decay. Collisions with other helium atoms in the target mixed the Rydberg ionic states with low $-\ell$ states at high $n$ which have large overlap with the nucleus. The charged pions emerging from the antiproton annihilations were detected by Cherenkov counters made of acryl [22] surrounding the target. In this way, the resonance condition between the laser beam and the atom was revealed as a sharp peak in the annihilation rate.

Figure 3 shows the resonance profiles of the transitions $(n, \ell)=(36,34) \rightarrow(37,33)$ in $\bar{p}^{3} \mathrm{He}^{+}$, and $(37,35) \rightarrow(38,34)$ and $(36,34) \rightarrow(35,33)$ in $\bar{p}^{4} \mathrm{He}^{+}$measured in this way [4]. The two peaks separated by $1.8 \mathrm{GHz}$ in Fig. 3a-b arise from the dominant interaction between the orbital angular momentum of the antiproton and electron spin. Actually the $\bar{p}^{3} \mathrm{He}^{+}$and $\bar{p}^{4} \mathrm{He}^{+}$states $(n, \ell)$ are respectively split into eight and four hyperfine substates due to the spin-spin interactions of the constituent particles [8]. The spectra of Fig. 3a thus contains (1) eight intense lines (indicated by four arrowed pairs) corresponding to E1 transitions involving no spin-flip, and (2) 12 weak lines wherein one of the constituent particles flips its spin. These closely-spaced sublines however cannot be resolved due to the $400-\mathrm{MHz}$ Doppler broadening caused by the motion of the $\bar{p} \mathrm{He}^{+}$in the target at $T \sim 10 \mathrm{~K}$.

The transition frequency of the $\bar{p}^{4} \mathrm{He}^{+}$resonance $(37,35) \rightarrow(38,34)$ measured over the years using antiproton beams of energies between $70 \mathrm{keV}$ and $5 \mathrm{MeV}$, and 


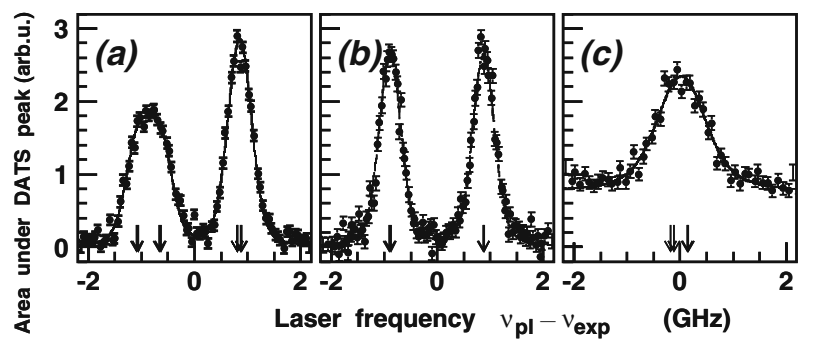

Fig. 3 Resonance profiles of the transition a $(n, \ell)=(36,34) \rightarrow(37,33)$ in $\bar{p}^{3} \mathrm{He}^{+}$, and b $(37,35) \rightarrow(38,34)$ and $\mathbf{c}(36,34) \rightarrow(35,33)$ in $\bar{p}^{4} \mathrm{He}^{+}$. The intensity of the laser-induced annihilation is shown as a function of the detuning of the laser frequency $v_{\mathrm{pl}}$ from the transition centroid $v_{\exp }$, see text

Fig. 4 a Frequency of the $\bar{p}^{4} \mathrm{He}^{+}$transition $(37,35) \rightarrow(38,34)$ measured in three experiments [2-4] compared with $\mathbf{b}$ the result of a three-body QED calculation [7]. Proton-to-electron [9] mass ratios according to the CODATA 98 and 2002 compilations, compared with the antiproton-to-electron mass ratio obtained in this experiment (a)

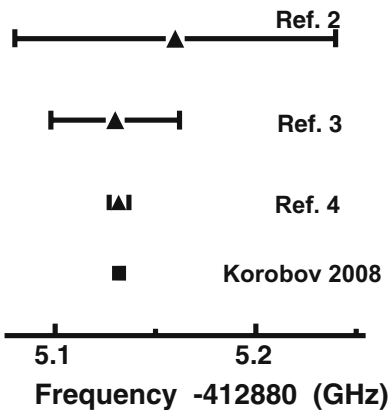

(b)

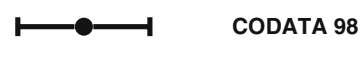

ம) CODATA 02

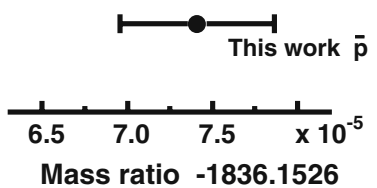

various types of lasers are shown in Fig. 4a. The results agree within $<1 \times 10^{-8}$ with theoretical calculations which claim a precision of $1-2 \mathrm{MHz}$ [7]. This calculation used as inputs the mass ratios between the proton, ${ }^{4} \mathrm{He}$ and ${ }^{3} \mathrm{He}$ nuclei, and electron [9]. We next determined the antiproton-to-electron mass ratio $M_{\bar{p}} / m_{e}=1836.152674$ (5) which results in theoretical values of the $\bar{p} \mathrm{He}^{+}$transition frequencies that agree best with experiments. More details can be found in [4] and [1].

\section{Continuous-wave pulse-amplified dye lasers}

It is generally difficult to stabilize the optical frequency $v_{\mathrm{pl}}$ of nanosecond lasers. This is because $v_{\mathrm{pl}}$ is modulated by nanosecond-scale changes in the refractive index $n_{c}$ of the gain media during the laser amplification. This causes a modulation or "chirp" relative to the seed laser frequency $v_{\mathrm{cw}}$,

$$
\Delta v_{c}=v_{\mathrm{pl}}-v_{\mathrm{cw}}=-\frac{1}{2 \pi} \frac{d \phi}{d t} \propto \frac{d n_{c}}{d t},
$$

wherein $\phi$ denotes the optical phase. These effects can broaden the laser linewidth and shift its frequency by several tens $\mathrm{MHz}$ or more, especially in high-power lasers involving large values of $d n_{c} / d t$. It is therefore vital in any high precision spectroscopy experiment to characterize the chirp $\Delta v_{c}$. 


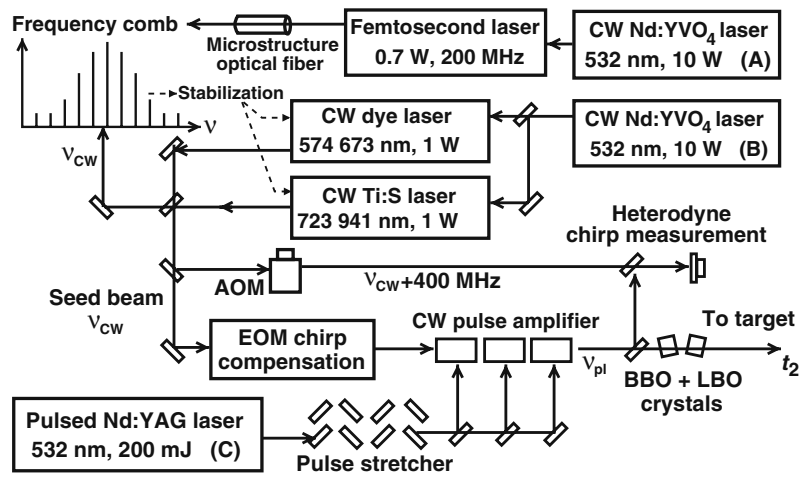

Fig. 5 Dye laser system used for spectroscopy of $\bar{p} \mathrm{He}^{+}$. Single-line-mode cw dye and Ti:S lasers were locked to a femtosecond frequency comb. This seed beam was amplified to a pulse energy of $E=5-20 \mathrm{~mJ}$ using three dye cells pumped by a Nd:YAG laser. The frequency chirp induced in the dye cells were measured by a heterodyne spectrometer, and corrected using an electro-optic modulator (EOM). In this way, a linewidth of $\sim 60 \mathrm{MHz}$ was achieved in the pulsed laser

We circumvented some of these problems [4] by basing our spectroscopy on continuous-wave (cw) dye and titanium sapphire (Ti:S) lasers [4]. These lasers of output power $P \sim 1 \mathrm{~W}$ and linewidth $\Gamma=0.1-1 \mathrm{MHz}$ [Fig. 5] were readily stabilized with a precision of $\sim 200 \mathrm{kHz}$ against one of the modes of a Ti:S femtosecond frequency comb $[23,24]$. The $\mathrm{cw}$ laser frequency was then scanned by changing the repetition rate of the femtosecond laser. The $\mathrm{cw}$ seed beam was amplified to a pulse energy $E=5-20 \mathrm{~mJ}$ in three dye cells pumped by a Nd:YAG laser. Betabarium borate $(\mathrm{BBO})$ and lithium triborate $(\mathrm{LBO})$ crystals were used to frequency double or triple the laser beam to wavelengths $\lambda=264.7-470.7 \mathrm{~nm}$. We measured the frequency chirp $\Delta v_{c}$ by superimposing the pulsed and cw seed beams on a photodiode and measuring the heterodyne beat signal. An electro-optic modulator (EOM) was used to apply a frequency shift to the seed laser which canceled the chirp $\Delta v_{c}[25,26]$. The linewidth of the pulsed laser beam was typically around $\Gamma \sim 60 \mathrm{MHz}[4]$.

\section{Seeded titanium-sapphire laser}

Nanosecond dye amplifiers typically generate laser pulses of $\Delta t<20$ ns with spectral linewidth greater than $\Gamma=50-100 \mathrm{MHz}$. To achieve smaller linewidths needed for future $\bar{p} \mathrm{He}^{+}$spectroscopy experiments, we developed [27] a Ti:S ring laser of circumference $800 \mathrm{~mm}$ (Fig. 6). This oscillator generated longer $(\Delta t=40-100 \mathrm{~ns})$ laser pulses of energy $E \sim 8-15 \mathrm{~mJ}$ and output wavelength $\lambda=726-941 \mathrm{~nm}$ [27]. The beam was amplified to $E \sim 50-100 \mathrm{~mJ}$ by making 3 passes through another Ti:S crystal (indicated by C2). The ring cavity consisted of a concave mirror (M1), a flat output coupler (OC) of reflectivity $R=80-90 \%$, and a prism which coarsely determined the laser wavelength. A pair of EOM crystals made of potassium dideuterium phosphate cut at Brewster's angle was placed between OC and the prism to carry out the chirp compensation. The $\mathrm{cw}$ seed laser beam traversed two Faraday isolators (FI1 and FI2), a single-mode fiber, and some lenses L1-L3 before 
Fig. 6 Layout of the

chirp-compensated Ti:S laser. The cw seed light is amplified to a pulse energy of 8-15 $\mathrm{mJ}$ using a triangular oscillator. The beam is then amplified to 50-100 mJ by making 3 passes through another Ti:S crystal. An electro-optic modulator is used to correct the frequency chirp. Abbreviations defined in the text

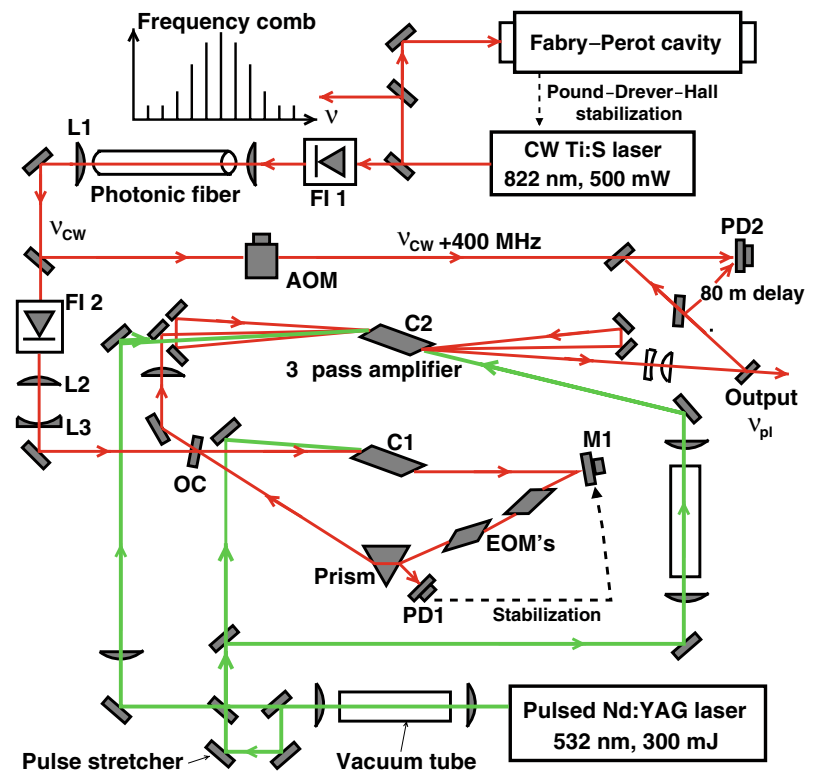

being coupled into the pulsed Ti:S cavity through mirror OC. The power circulating in the cavity was monitored using a photodiode. The cavity length was locked to the seed beam to maximize this. To prevent the strong pulsed light from disturbing this lock, a track-and-hold regulator froze the correction signal $\sim 100 \mu$ s before the pumping [27].

The time evolutions of the intensity and chirp of the laser pulse are shown in Fig. 7a-b. The frequency of the uncorrected laser (indicated by the broken line) shifted to $\Delta v_{c}(t)<-10 \mathrm{MHz}$ before returning to zero during the laser pulse. We carried out Doppler-free two-photon spectroscopy of the $F=4$ hyperfine component in the $6 s_{1 / 2}-8 s_{1 / 2}$ two-photon transition in Cs using this laser beam [27]. The observed Cs line [Fig. 7c] was correspondingly asymmetric and its centroid shifted by $-10 \mathrm{MHz}$ relative to $v_{\mathrm{cw}}$, whereas its linewidth $\Gamma_{\mathrm{Cs}} \sim 15 \mathrm{MHz}$ was much larger than the Fourier limit suggested by Fig. 7a.

Next, the chirp was corrected to $\left|\Delta v_{c}(t)\right| \leq 2 \mathrm{MHz}$ over most of the laser pulse [solid line in Fig. 7b] using the EOM. The laser linewidth was now inferred to be nearly equal to the Fourier limit $\Gamma_{\mathrm{pl}} \sim 6 \mathrm{MHz}$. The observed Cs resonance [Fig. 7d] was 3 times narrower $\left(\Gamma_{\mathrm{Cs}} \sim 4.5 \mathrm{MHz}\right)$ than in the uncorrected case, whereas its centroid is $\Delta v_{c} \sim 0$. We calculated the expected Cs lineshape by integrating twophoton optical rate equations. We included effects due to the chirp and temporal and spatial profiles of the laser pulse. By fitting the data with this theoretical curve, we determined the resonance centroid as $v_{6 \mathrm{~s}-8 \mathrm{~s}}(F=4)=364,503,080.3(5) \mathrm{MHz}$ [27]. This measurement with a precision of 1.4 parts per $10^{9}$ is in good agreement with published values [29] of much higher precision. The EOM chirp correction technique has thus resulted in one of the smallest linewidth $(6 \mathrm{MHz})$ reported so far for a Ti:S laser of output energy $E=50-100 \mathrm{~mJ}$. These laser techniques were also used to detect hydrogen gas leaks [28] from fuel cells. 
Fig. 7 Time evolution of the intensity (a) and frequency chirp (b) of Ti:S laser pulses, with (solid line) and without (broken line) chirp compensation. The $6 \mathrm{~s}-8 \mathrm{~s}$ $(F=4)$ two-photon resonance of Cs as a function of $v_{\mathrm{cw}}$, without (c) and with (d) compensation
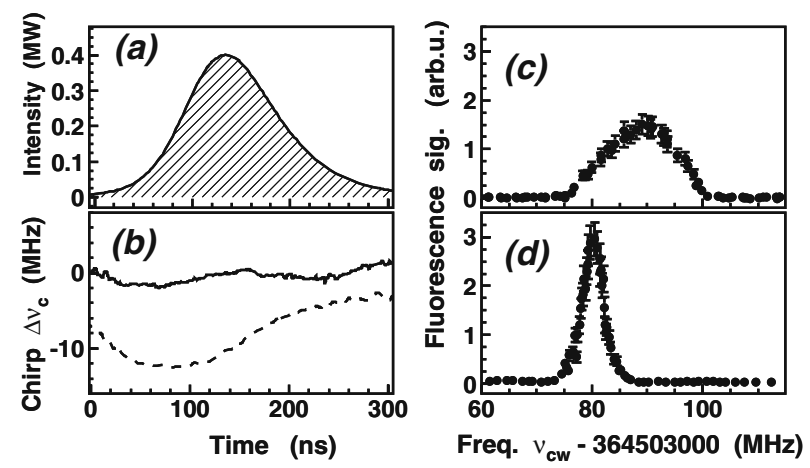

\section{Particle detectors}

In these laser spectroscopy experiments, it was crucial to measure the beam profile of the antiproton beam entering the helium target, and ensure a good spatial overlap with the laser beam (see Fig. 2). For this we are currently developing several types of profile monitors [30-32] wherein the antiproton beam is intercepted by some segmented electrode foils or grids of wires arranged in XY configurations. One design [31] used preamplifiers to detect the charge induced by secondary electrons escaping from each foil segment or wire, thereby measuring the $\mathrm{X}$ - and $\mathrm{Y}$ - spatial projections of the beam profile with a resolution of $0.25-1 \mathrm{~mm}$. The detector had a root-mean-square noise charge (i.e., the signal charge for which the signal-tonoise ratio is unity) of around $\varepsilon \sim 200$ electrons. This is within a factor 2 of the best values reported for any room-temperature detector of comparable $\left(C_{d} \sim 10\right.$ $\mathrm{pF})$ capacitance. The detectors were used in ultrahigh vacuum $\left(p \sim 10^{-8} \mathrm{~Pa}\right)$, low temperatures $(T<100 \mathrm{~K})$, and magnetic fields of $B>0.1 \mathrm{~T}$.

One disadvantage of these profile monitors is that their readout electronics were large and expensive, and they consumed many hundreds of watts of power. The preamplifiers were manufactured by soldering hundreds of junction field-effect transistors (JFETs) on hybrid circuit boards [31] and carefully adjusting e.g. their gain using trimmer resistors. We are now developing detectors which utilize very large scale integration (VLSI) circuits manufactured by complementary metal oxide semiconductor (CMOS) technology. The sensitivity of these CMOS preamplifiers are typically worse compared to JFET ones at $C_{d} \sim 10 \mathrm{pF}$, but simulations indicate that a clear beam profile would still be observed.

By using the improved lasers and detectors described here, we intend to carry out laser spectroscopy experiments of $\bar{p} \mathrm{He}^{+}$with parts-per-billion scale precisions in the near future. This may allow us to verify the CPT symmetry via comparison of the antiproton and proton masses and charges with a similar level of precision.

Acknowledgements I am deeply indebted to the ASACUSA collaboration. This work was supported by the European Young Investigator (EURYI) award of the European Science Foundation and the Deutsche Forschungsgemeinschaft (DFG), the Munich Advanced Photonics (MAP) cluster of DFG, and the Grant-in-Aid for Specially Promoted Research (20002003) of MEXT, Japan. 
Open Access This article is distributed under the terms of the Creative Commons Attribution Noncommercial License which permits any noncommercial use, distribution, and reproduction in any medium, provided the original author(s) and source are credited.

\section{References}

1. Hayano, R.S., Hori, M., Horváth, D., Widmann, E.: Antiprotonic helium and CPT invariance. Rep. Prog. Phys. 70, 1995 (2007)

2. Hori, M., et al:: Sub-ppm laser spectroscopy of antiprotonic helium and a CPT-violating limit on the antiprotonic charge and mass. Phys. Rev. Lett. 87, 093401 (2001)

3. Hori, M., et al.: Direct measurement of transition frequencies in isolated $\bar{p} \mathrm{He}^{+}$atoms, and new CPT violation limits on the antiproton charge and mass. Phys. Rev. Lett. 91, 123401 (2003)

4. Hori, M., et al.: Determination of the antiproton-to-electron mass ratio by precision laser spectroscopy of $\bar{p} \mathrm{He}^{+}$. Phys. Rev. Lett. 96, 243401 (2006)

5. Korobov, V.I.: Metastable states in the antiprotonic helium atom decaying via Auger transitions. Phys. Rev., A 67, 062501 (2003)

6. Kino, Y., Kamimura, M., Kudo, H.: Numerical accuracy of the energy levels of antiprotonic helium atoms. Nucl. Instrum. Methods, B 214, 84 (2004)

7. Korobov, V.I.: Calculation of transitions between metastable states of antiprotonic helium including relativistic and radiative corrections of order $R_{\infty} \alpha^{4}$. Phys. Rev., A 77, 042506 (2008)

8. Korobov, V.I.: Hyperfine structure of metastable states in ${ }^{3} \mathrm{He}^{+} \bar{p}$ atom. Phys. Rev., A 73, 022509 (2006)

9. Mohr, P.J., Taylor, B.N., Newell, D.B.: Rev. Mod. Phys. 80, 633 (2008)

10. Gabrielse, G., et al.: Antiproton confinement in a Penning-Ioffe trap for antihydrogen. Phys. Rev. Lett. 98, 113002 (2007)

11. Andresen, G., et al.: Antimatter plasmas in multipole trap for antihydrogen. Phys. Rev. Lett. 98, 023402 (2007)

12. Testera, G., et al.: Formation of a cold antihydrogen beam in AEGIS for gravity measurements. AIP Conf. Proc. 1037, 5 (2008)

13. Lombardi, A.M., Pirkl, W., Bylinsky, Y.: In: Proceedings of the 2001 particle accelerator conference, Chicago, pp. 585-587 (2001)

14. Hori, M., et al.: Primary populations of metastable antiprotonic ${ }^{4} \mathrm{He}$ and ${ }^{3} \mathrm{He}$ atoms. Phys. Rev. Lett. 89, 093401 (2002)

15. Hori, M., et al:: Laser spectroscopic studies of state-dependent collisional quenching of the lifetimes of metastable antiprotonic helium atoms. Phys. Rev., A 57, 1698 (1998)

16. Hori, M., et al.: Laser spectroscopic studies of state-dependent collisional quenching of the lifetimes of metastable antiprotonic helium atoms. Phys. Rev., A 58, 1612 (1998)

17. Hori, M., et al.: Populations and lifetimes in the $v=n-l-1=2$ and 3 metastable cascades of $\bar{p} \mathrm{He}^{+}$measured by pulsed and continuous antiproton beams. Phys. Rev., A 70, 012504 (2004)

18. Hori, M., Hayano, R.S., Widmann, E., Torii, H.A.: Resolution enhancements of the $\bar{p} \mathrm{He}^{+}$atomic line profiles measured with a pulsed dye laser and Fizeau wavelength meter. Opt. Lett. 28, 2479 (2003)

19. Yamaguchi, H., et al.: Anomalies in the decay rates of antiprotonic helium atom states. Phys. Rev., A 66, 022504 (2002)

20. Yamaguchi, H., et al.: Systematic study of the decay rates of antiprotonic helium states. Phys. Rev., A 70, 012501 (2004)

21. Hori, M., et al.: Observation of cold, long-lived antiprotonic helium ions. Phys. Rev. Lett. 94, 063401 (2005)

22. Hori, M., Yamashita, K., Hayano, R.S., Yamazaki, T.: Analog Cherenkov detectors used in laser spectroscopy experiments on antiprotonic helium. Nucl. Instrum. Methods Phys. Res., A 496, 102 (2003)

23. Udem, Th., Holzwarth, R., Hänsch, T.W.: Optical frequency metrology. Nature 416, 233 (2002)

24. Holzwarth, R., et al.: Optical frequency synthesizer for precision spectroscopy. Phys. Rev. Lett. 85, $2264(2000)$

25. Eikema, K.S.E., Ubachs, W., Vassen, W., Hogervorst, W.: Lamb shift measurement in the $1^{1} \mathrm{~S}$ ground state of helium. Phys. Rev., A 55, 1866 (1997) 
26. Meyer, V., et al.: Measurement of the 1s-2s energy interval in muonium. Phys. Rev. Lett. 84, $1136(2000)$

27. Hori, M., Dax, A.: Chirp-corrected, nanosecond Ti:sapphire laser with $6 \mathrm{MHz}$ linewidth for spectroscopy of antiprotonic helium. Opt. Lett. 34, 1273 (2009)

28. Hori, M., Hayano, R.S., Fukuta, M., Koyama, T., Nobusue, H., Tanaka, J.: Large-area imager of hydrogen leaks in fuel cells using laser-induced breakdown spectroscopy. Rev. Sci. Instrum. 80, 103104 (2009)

29. Fendel, P., Bergeson, S.D., Udem, Th., Hänsch, T.W.: Two-photon frequency comb spectroscopy of the $6 \mathrm{~s}-8 \mathrm{~s}$ transition in cesium. Opt. Lett. 32, 701 (2007)

30. Hori, M., Hanke, K.: Spatial and temporal beam profile monitor with nanosecond resolution for CERN's linac4 and superconducting proton linac. Nucl. Instrum. Methods, A 588, 359 (2008)

31. Hori, M.: Photocathode microwire monitor for nondestructive and highly sensitive spatial profile measurements of ultraviolet, x-ray, and charged particle beams. Rev. Sci. Instrum. 76, 113303 (2005)

32. Hori, M.: Parallel plate chambers for monitoring the profiles of high-intensity pulsed antiproton beams. Nucl. Instrum. Methods Phys. Res., A 522, 420 (2004) 